\title{
Note on Transliteration, Translation, and Dates
}

Whenever possible, the transliteration of Russian names and titles complies with the British Standard System, modified to omit hard and soft signs. The only exceptions to that rule pertain to familiar names that have acquired a recognized English spelling, and to material quoted from sources using other transliteration systems. Unless specified, translations are by the author.

In February 1918, Russia shifted from the Julian to the Gregorian calendar. Unless specified, all dates before February 1918 refer to the Julian calendar, while those after February 1918 refer to the Gregorian calendar. 
This page intentionally left blank 